\title{
99mTc-HMPAO-Labeled WBC Scan for the Diagnosis of Chronic Recurrent Multifocal Osteomyelitis
}

\author{
Tyler A. Dailey, Michael D. Berven, and Penny J. Vroman
}

Department of Radiology, San Antonio Military Medical Center, Fort Sam Houston, Texas

The goal of this teaching case study is to demonstrate the novel use of $99 \mathrm{mTc}$-HMPAO-labeled white blood cells (WBCs) in diagnosing chronic recurrent multifocal osteomyelitis (CRMO). 99mTc-HMPAO-labeled white blood cells were utilized to diagnose CRMO. Classically, CRMO is evaluated scintigraphically using 99mTc-methylene diphosphonate/hydroxymethylene diphosphonate. ${ }^{99 m}$ Tc-HMPAO-labeled WBCs were chosen over ${ }^{111} \mathrm{In}$-labeled WBCs because of the former's improved imaging characteristics and decreased radiation dose. ${ }^{99 m T c-}$ HMPAO-labeled WBCs were successful in diagnosing CRMO. $99 \mathrm{~m}$ Tc-HMPAO-labeled WBC scanning is specific for the diagnosis of CRMO.

Key Words: bone scan; chronic recurrent multifocal osteomyelitis; HMPAO; pediatric

J Nucl Med Technol 2014; 42:299-301

DOI: 10.2967/jnmt.114.138073

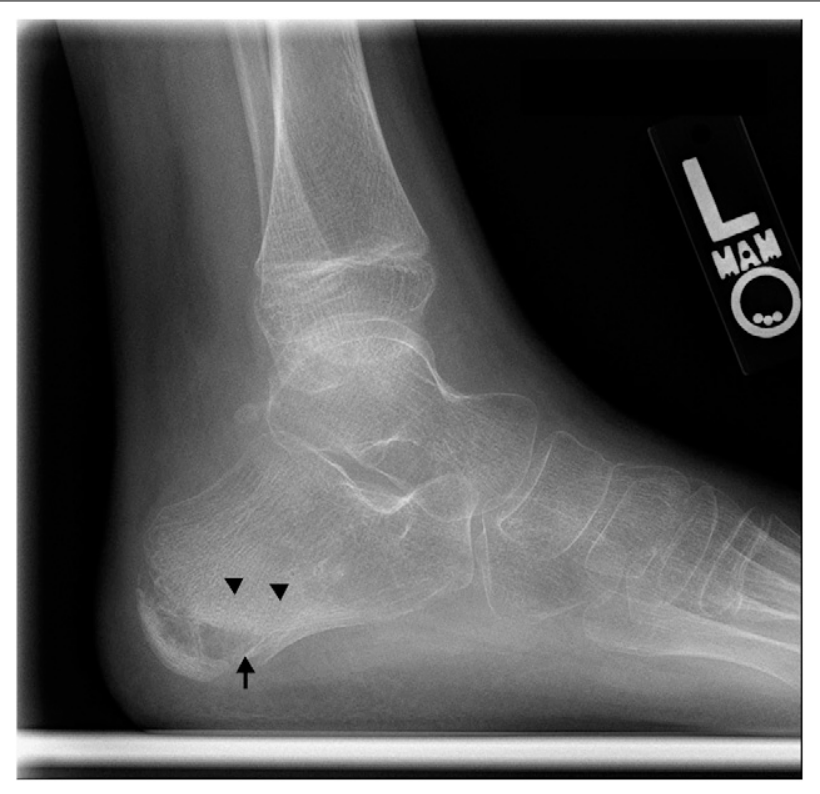

FIGURE 1. Radiograph obtained 3 mo after onset of symptoms shows osteolysis (arrow) with surrounding sclerosis (arrowheads).

Received Jan. 25, 2014; revision accepted Apr. 15, 2014.

For correspondence or reprints contact: Tyler A. Dailey, 3551 Roger Brooke Dr., Department of Radiology, Attn: Residency Program Coordinator, Fort Sam Houston, TX 78234-4504.

E-mail: tyler.a.dailey2.mil@mail.mil

Published online Aug. 28, 2014.

COPYRIGHT (C) 2014 by the Society of Nuclear Medicine and Molecular Imaging, Inc.
W present a case illustrating the novel use of ${ }^{99 \mathrm{~m}} \mathrm{Tc}$ hexamethylpropyleneamine oxime (HMPAO)-labeled white blood cells (WBCs) in diagnosing chronic recurrent multifocal osteomyelitis (CRMO). The 99m Tc-HMPAO WBCs were instrumental in confirming the inflammatory nature of the patient's complaint and in identifying unsuspected sites of disease, typical of CRMO. The ${ }^{99 \mathrm{~m}}$ Tc-HMPAO WBC findings of CRMO were confirmed by pathologic analysis and clinical follow-up. We believe this is the first case to illustrate the utility of ${ }^{99 \mathrm{~m}} \mathrm{Tc}-\mathrm{HMPAO}$ WBCs in diagnosing CRMO.

\section{CASE REPORT}

A 10-y-old boy presented with atraumatic left heel pain and swelling. Initial radiographs showed calcaneal sclerosis and apophyseal irregularity (Fig. 1). Stress injury was diagnosed. Despite escalating treatment, symptoms continued. After $5 \mathrm{mo}$, MR imaging was performed and demonstrated edema and enhancement but no fluid collection, mass, or sequestration (Fig. 2). A pediatric orthopedist was consulted, who raised concern about chronic

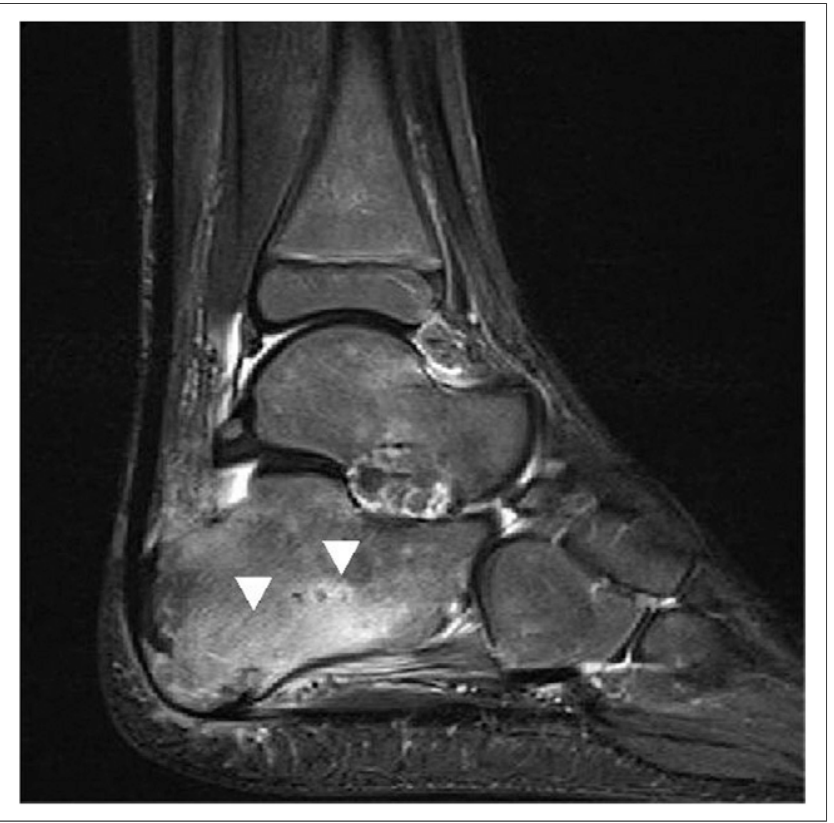

FIGURE 2. Sagittal short- $\tau$ inversion recovery MR image demonstrates bone marrow edema in calcaneus (arrowheads). Small amount of edema is seen in surrounding soft tissues 
osteomyelitis versus the less likely osteoid osteoma or chondroblastoma.

A labeled WBC study was obtained to evaluate for infection. Instead of ${ }^{111} \mathrm{In}$, ${ }^{99 \mathrm{~m}} \mathrm{Tc}-\mathrm{HMPAO}$ was chosen because of its better dosimetry - a major concern in pedi-

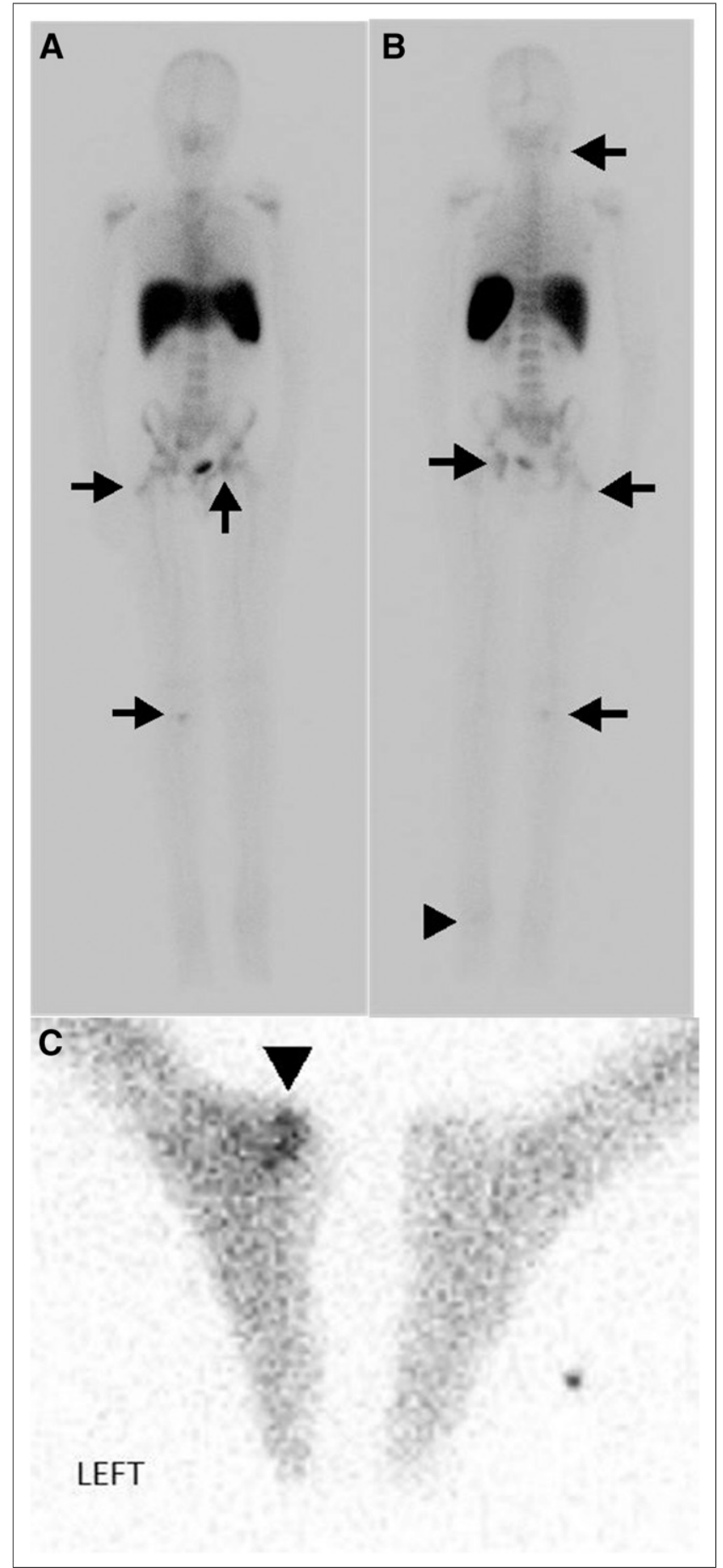

FIGURE 3. Anterior (A) and posterior (B) whole-body planar images and spot image (C) from 99mTc-HMPAO WBC scan show increased uptake at left calcaneus (arrowhead). Additional radiotracer uptake is seen in right tibial plateau, left acetabulum, right greater trochanter, and right mandible (arrows). None of these sites were symptomatic.

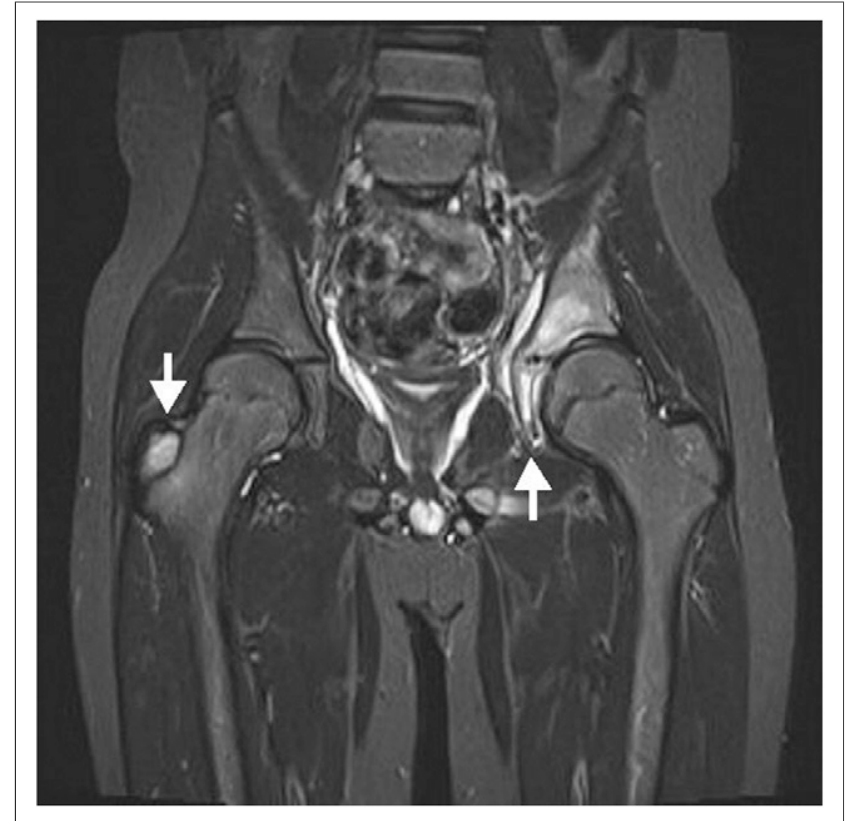

FIGURE 4. Coronal short- $\tau$ inversion recovery MR image of pelvis demonstrates bone marrow edema in left acetabulum and right greater trochanter (arrows) corresponding to sites of asymptomatic WBC accumulation.

atric patients. The examination showed abnormal activity in the calcaneus. There was also abnormal activity at several asymptomatic skeletal sites (Fig. 3). MR imaging of the pelvis was performed to assess for possible pelvic soft-tissue involvement (1). The MR imaging showed edema and enhancement in the left acetabulum and in the right greater trochanter (Fig. 4).

The patient underwent open biopsy of the calcaneus. No organisms were identified and cultures were negative. Tissue analysis demonstrated inflammation, to include suppurative granulomata. CRMO was diagnosed and therapy with nonsteroidal antiinflammatory drugs was initiated, with the patient having a positive response.

\section{DISCUSSION}

CRMO is an inflammatory skeletal disorder of unknown etiology that remains a diagnosis of exclusion (2). It is predominately a pediatric disease; the mean age of patients at presentation is $10.5 \mathrm{y}$. CRMO typically presents with an insidious onset of unifocal pain and swelling (3). Long bone metaphases are most frequently involved, but the clavicles, pelvis, spine, and calcanei may also be affected (4). The radiographic appearance can be lytic, sclerotic, or mixed (5). MR imaging will demonstrate marrow edema without abscess. The primary differential considerations for diagnosis of CRMO include tumors such as metastases, Ewing sarcoma, osteoid osteoma, chondroblastoma, and eosinophilic granuloma.

Classically, CRMO is evaluated scintigraphically using 99mTc-methylene diphosphonate/hydroxymethylene diphosphonate, which shows increased osteoblastic activity at the site of 
concern and identifies sites of asymptomatic disease (4). Multifocality is characteristic, and a study by Mandell et al. demonstrated that patients have a mean of 6 lesions on bone scans (5). The use of labeled WBCs for diagnosing CRMO has not previously been described but was chosen in this case for its specificity in identifying infection and inflammation. Labeling with ${ }^{99 \mathrm{~m} T c-H M P A O}$ instead of ${ }^{111}$ In was chosen for the former's improved imaging characteristics and decreased radiation dose. Like bone scintigraphy, ${ }^{99 \mathrm{~m} T c-H M P A O ~ W B C s}$ identified asymptomatic foci, critical to the diagnosis of CRMO.

Histologic evaluation demonstrates an inflammatory process with occasional granulomatous foci, but no organisms (6). Treatment is typically with nonsteroidal antiinflammatory drugs and, more recently, tumor necrosis factor $\alpha$ inhibitors (2).

\section{CONCLUSION}

99m Tc-HMPAO-labeled WBC scanning is specific for the diagnosis of infection and inflammation, including CRMO, and helpful for differentiation from neoplastic entities. ${ }^{99 \mathrm{~m}} \mathrm{Tc}-\mathrm{HMPAO}$ is preferred over ${ }^{111} \mathrm{In}$ in the pediatric population because of the decreased radiation dose.

\section{DISCLOSURE}

No potential conflict of interest relevant to this article was reported.

\section{REFERENCES}

1. Treves ST. Pediatric Nuclear Medicine/PET. New York, NY: Springer; 2007.

2. Wipff J, Adamsbaum C, Kahan A, Job-Deslandre C. Chronic recurrent multifocal osteomyelitis. Joint Bone Spine. 2011;78:555-560.

3. Iyer RS, Thapa MM, Chew FS. Chronic recurrent multifocal osteomyelitis. AJR. 2011;196(suppl):S87-S91.

4. Khanna G, Sato TS, Ferguson P. Imaging of chronic recurrent multifocal osteomyelitis. Radiographics. 2009;29:1159-1177.

5. Mandell GA, Contreras SJ, Conard K, Harcke T, Maas KW. Bone scintigraphy in the detection of chronic recurrent multifocal osteomyelitis. J Nucl Med. 1998; 39:1778-1783.

6. Mortensson W, Edeburn G, Fries M, Nilsson R. Chronic recurrent multifocal osteomyelitis in children: a roentgenologic and scintigraphic investigation. Acta Radiol. 1988;29:565-570. 